\title{
MAGNETIC FIELD IN THE SUN'S INTERIOR FROM OSCILLATION DATA
}

\author{
W.A.DZIEMBOWSKI \\ Copernicus Astronomical Center \\ Warszawa, Poland \\ P.R.GOODE \\ Department of Physics, N.J.I.T. \\ Newark, NJ, USA
}

\begin{abstract}
Solar oscillations provide a probe of the internal magnetic field of the Sun if the field has sufficient intensity. Using the oscillation data of Libbrecht, we find evidence for a $2 \pm 1$ megagauss quadrupole toroidal field centered at 0.7 of the solar radius which is barely beneath the base of the convection zone. This field, by its location and symmetry, may be associated with the dynamo that drives the Sun's 22-year activity cycle.
\end{abstract}

\section{Introduction}

Helioseismology is the science by which solar oscillations are used to determine internal properties of the Sun. In particular, helioseismological techniques have yielded detailed information about the interior rotation of the Sun [Duvall, et al (1984), Duvall, Harvey and Pomerantz (1986), Brown, et al (1989), Christensen-Dalsgaard and Shou (1988), and Dziembowski, Goode, and Libbrecht (1989)] and the radial dependence of the speed of sound [Christensen-Dalsgaard, et al (1985) and Brodsky and Vorontsov (1988)]. It is our purpose here to discuss the use of solar oscillations to extract information about the Sun's internal magnetic field. In the long run, the study of solar oscillations is our only real hope for determining the internal magnetism of the Sun.

Before the advent of more detailed solar oscillation data, the size of the magnetic field throughout the radiative interior was more open to question. At one extreme, Ulrich and Rhodes (1985) assumed a relic field of $300 \mathrm{MG}$, centered in the core, in an effort to describe some gross structures in oscillation data. At the other extreme, Spruit (1987) has argued that magnetic torques would preclude a steady-state field of more than a few Gauss in the interior.

Recent oscillation data like those due to Duvall, Harvey and Pomerantz (1986), Brown and Morrow (1987), and Libbrecht (1989) have shed some light on this situation. These data best sample the region near the base of the convection zone ( 0.73 of the radius). From these data, Brown, et al (1989), Christensen-Dalsgaard and Shou (1988) and Dziembowski, Goode, and Libbrecht (1989) calculated rotation laws which show no significant gradient above the base of the convection zone. Rather, near the base of the convection zone, there is a fairly sharp transition from surface-like differential rotation just above to solid body-like rotation just 
beneath. Brown, et al (1989) used this result to suggest that the dynamo driving the 22-year activity cycle is seated just beneath the convection zone. Since differential rotation may persist into the outer radiative zone, there one may naively expect that a small poloidal field could be sheared into a sizeable quadrupole toroidal field. In fact, Dziembowski and Goode (1989) used the data of Libbrecht (1989) to calculate such a toroidal field of amplitude $2 \pm 1 \mathrm{MG}$ centered just beneath the convection zone. This field may be associated with the dynamo since it has the proper symmetry and proximity. However, the calculated field is two orders of magnitude more intense than generally expected for the dynamo field [Parker (1987)]. Since the $10^{4} \mathrm{G}$ dynamo field is expected at the base of the convection zone, the calculated field could be a slightly deeper lying reservoir for the dynamo. We shall see that that oscillation data can be used, as well, to place limits on the toroidal field considerably deeper than the base of the convection zone.

A megagauss toroidal field fed by differential rotation is not in steady state and, therefore, is not precluded by Spruit's argument. Kuhn (1988) has argued that the symmetric part of the oscillation spectrum changes over the 22-year solar activity cycle. If he were correct, synchronous, large variations in the calculated field would be a source of the changes.

\section{Solar Oscillations in the Presence of a Magnetic Field}

Global solar oscillations are sound waves which sample the interior of the Sun. An individual oscillation is characterized by the product of a radial part, labelled by $n$ the radial order, and a single $Y_{\ell}^{m}(\vartheta, \varphi)$, where $\ell$ is it angular degree and $m$ is it azimuthal order. In the absence of a perturbing force the $(n, \ell)$-multiplets are $(2 \ell+1)$-fold degenerate in $m$. A magnetic field lifts this degeneracy. For an axially symmetric field, the structure of each $(n, \ell)$-multiplet is described by a polynomial in $\mathrm{m}^{2}$ having its order equal to the multipole order of the field. The coefficients of this polynomial are a quadratic function in, separately, the poloidal and toroidal field components. Consistent with the oscillation data, it will be assumed here that the axis of symmetry of the field is aligned with the rotation axis. Then, contributions to the coefficients come from the quadratic and higher effects of rotation, as well.

\section{Data}

We used the data obtained by Libbrecht (1989). His frequency splittings, $\nu_{n \ell m}-\nu_{n \ell 0}$, are given in terms of the $a_{i}$-coefficients from

$$
\nu_{n \ell m}-\nu_{n \ell 0}=\ell \sum_{i=1}^{5} a_{i} P_{i}\left(\frac{m}{\ell}\right),
$$

where $P_{i}$ is a Legendre polynomial. This Legendre expansion is a convenient way of representing the power series in $m$ which includes the $m^{2}$ terms discussed in the previous section. The data set covers the $\ell$-range $10-60$ and the frequency range $1.5-4.0 \mathrm{mHz}$. These data best sample the region between $x=0.5$ and 0.9 , where $x=r / R$. Lower degree oscillations would sample more deeply and higher degree oscillations would provide the contrast 




(a)

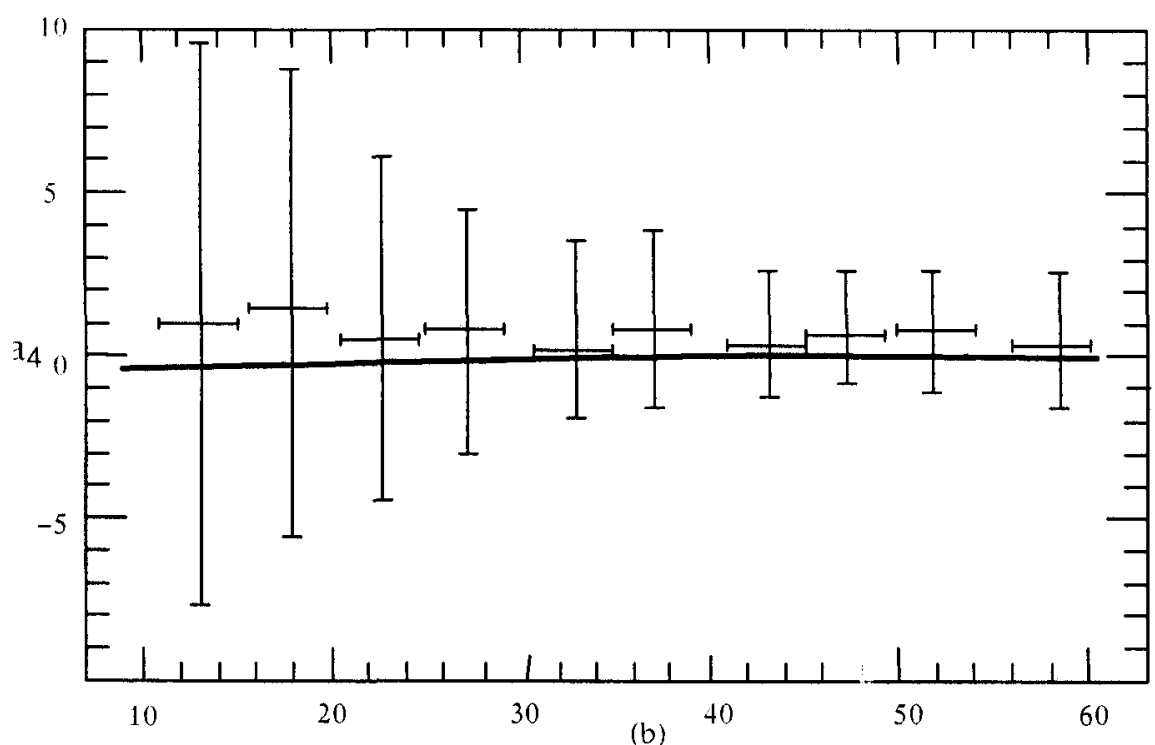

Figure 1. Weighted averages of Libbrecht's $\mathrm{a}_{2}-$ and $\mathrm{a}_{4}-$ coefficients $(n \mathrm{~Hz})$ vs 1 . The solid lines represent the calculated effect of distortion. 
to probe closer to the surface. Libbrecht's odd a-coefficients were used by ChristensenDalsgaard and Shou (1988) and Dziembowski, Goode and Libbrecht (1989) to calculate the internal rotation of the Sun as a function of radius and latitude from $x=0.4$ to 1.0. The even $a$-coefficients reflect centrifugal distortion and, for instance, an aligned axisymmetric magnetic field.

Figure 1 shows the measured values of the $a_{2}$ - and $a_{4}$-coefficients averaged over $n$ and grouped in bins $5 \ell$-values wide. The solid line represents the calculated effect of rotational distortion - this effect is subsequently subtracted from each multiplet. From the original data set covering 678 multiplets we removed 22 corresponding to the modes that could have been effected by an accidental degeneracy. We emphasize that if Libbrecht's data were averaged over $\mathrm{n}$, like the other available sets, we could not have determined a magnetic field from it.

\section{The Inverse Problem for a Toroidal Magnetic Field}

The aligned, axisymmetric toroidal magnetic field we assumed is given by

$$
B_{\varphi}^{2}=4 \pi p(x) \sin ^{2} \vartheta \sum_{k=1} \beta_{k}(x) \cos ^{2 k-2} \vartheta
$$

where $p(x)$ is the gas pressure. We assumed that the $\beta_{k}$ and their derivatives vanish at $x=0$ and 1 . The lowest order linear perturbation of the hydrostatic equation arising from the toroidal field yields our inverse problem

$$
a_{2 i}=\sum_{k \geq i} \int_{0}^{1}\left(\beta_{k} \mathbf{E}_{2 i, k}+x \frac{d \beta_{k}}{d x} \mathbf{D}_{2 i, k}\right) d x .
$$

The kernels $\mathbf{E}$ and $\mathbf{D}$ were determined from the mode eigenfunctions evaluated for a standard solar model. Examples of such kernels are shown in Figure 2 for $i=1$ and 2 and $k=2$. If we eliminate the $d \beta_{k} / d x$ - term by integrating by parts, the resulting kernel,

$$
\mathbf{F}_{2 i, k}=\mathbf{E}_{2 i, k}-\frac{d}{d x}\left(x \mathbf{D}_{2 i, k}\right)
$$

exhibits rapid sign changes - see the dashed lines shown in Figure 2. This latter property makes the kernel for each multiplet more orthogonal to those for the other multiplets. Since the success of the helioseismology depends critically on a differential sampling of the interior, the rapid sign changes greatly increase the prospects for the seismology. The inverse problem for the field closely resembles the one for differential rotation. Having two sets of even-a coefficients, we could have attempted to first determine $\beta_{2}$ from the $a_{4}$-coefficients and, subsequently, $\beta_{1}$ from $\beta_{2}$ and the $a_{2}$-coefficients. We found, however, that within the errors the $\beta_{2}$-term suffices. In the following, we consider this term alone and drop the subscript $k$ from $\beta$ and $\mathbf{F}$. 


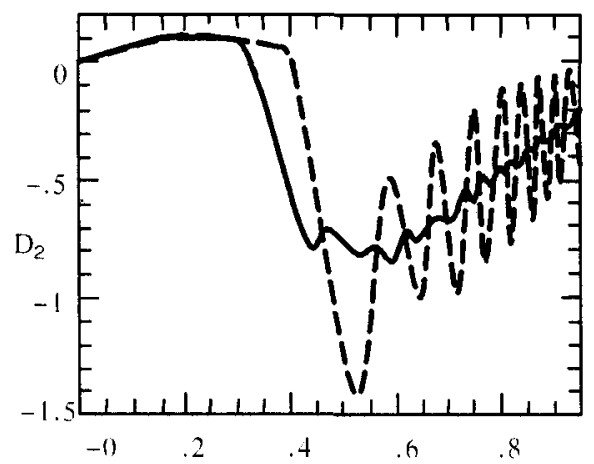

(a)

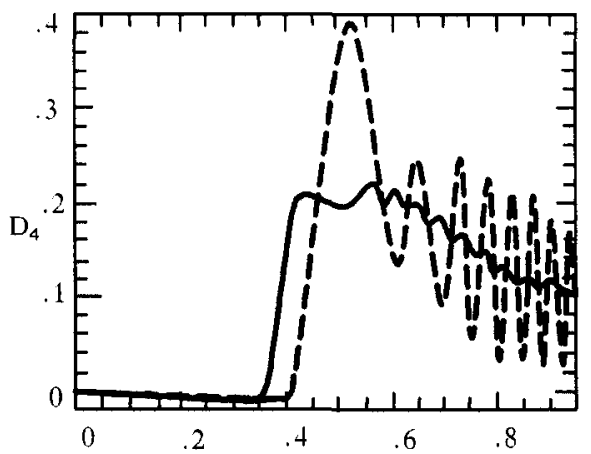

(c)

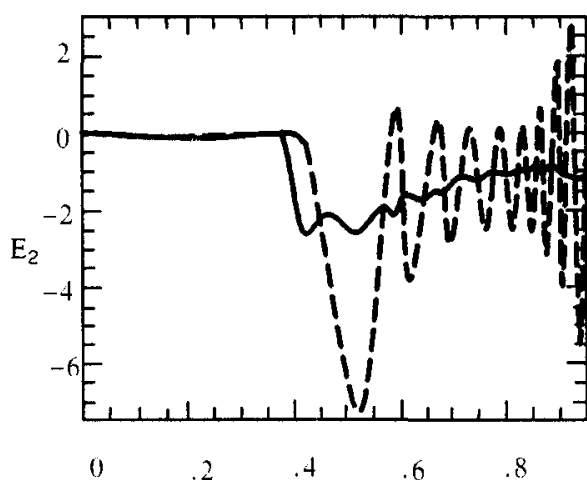

(b)

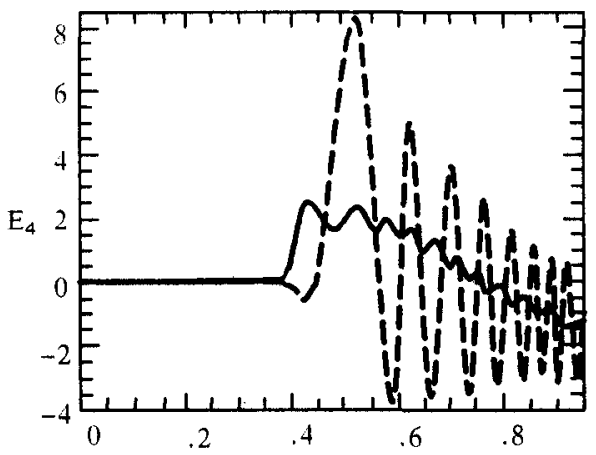

(d)

Figure 2. Sample a) $\mathrm{D}_{2}-$, b) $\mathrm{E}_{2}-$, c) $\mathrm{I}_{4}-$ and d) $\mathrm{E}_{4}-$ kernels vs. the fractional radius. The kernels are for $1=20$ and are given in $\mu \mathrm{Hz}$. The dashed line $(-\cdots)$ is for $n=14$ and the solid ( $)$ is for the average over $n$ in the five - minute period oscillation band. 


\section{Evidence for a Megagauss Magnetic Field Near the Base of the Solar Con- vective Envelope}

The inverse problem for the toroidal field was solved using two methods. Our primary approach employed the method of Backus and Gilbert (1970) in which a unimodular kernel, $\mathbf{K}$, is constructed from a linear combination of the $\mathbf{F}$ kernels which are calculated for each mode present in the data set. The linear combination must be localized at a selected point, $x_{0}$. We thus write

$$
\mathbf{K}_{2 i}\left(x, x_{0}\right)=\sum_{j} c_{2 i, j}\left(x_{0}\right) \mathbf{F}_{2 i, j}(x),
$$

where $j$ is the mode counter. The two sets of $c$-coefficients for each $x_{0}$ - one from the $a_{2}$ data and the other from the $a_{4}$-data - were determined by a compromise between maximal sharpness and minimal amplification of the errors from the data. If $\mathbf{K}$ is indeed sharp, one may expect that

$$
\beta\left(x_{0}\right)=\sum_{j} c_{2 i, j}\left(x_{0}\right) a_{2 i, j}
$$

However, if the field is more confined than the K-kernel sampling it, then the field will always be underestimated. The results for inversions at three selected locations are shown in Figure 3. Only the results for $x_{0}=0.7$ are significant, where we have

$$
\beta=(4.1 \pm 1.1) \times 10^{-3} \text { and }(4.9 \pm 1.7) \times 10^{-3}
$$

following from the $a_{2}$ - and $a_{4}$-data, respectively. These values correspond to a quadrupole toroidal field of amplitude $2 \pm 1 \mathrm{MG}$. At $x_{0}=0.55$ and 0.90 , the K-kernels are not so sharp and the average $\beta$-values are consistent with zero, however, the calculated errors in $\beta$ may be used to place limits on the average quadrupole toroidal field. At $x=0.55$, the limit is 4 $\mathrm{MG}$ and at $x=0.9$ the limit is $0.2 \mathrm{MG}$.

In the second method of inversion, we employed a discretization of $\beta(x)$ in terms of cubic spline functions and solved the inverse problem by a least squares method. Since the functional form of $\beta(x)$ was assumed, we calculated $d \beta / d x$ explicitly rather than performing an integration by parts. The significant results of the inversions occur at $x_{0}=0.7$ and are $\beta=(1.7 \pm 0.6) \times 10^{-3}$ and $(3.2 \pm 1.6) \times 10^{-3}$, respectively. These numbers are in reasonable agreement with those from the Backus-Gilbert method.

\section{Implications}

If a megagauss toroidal field were centered just beneath the convection zone, it would cause no observable dynamical effect. The ratio of the magnetic pressure to the gas pressure would be about $10^{-3}$ - having no perceptible effect on the speed of sound near the base of the convection zone. The quadrupole moment would be comparable to that which would result from a rigidly rotating Sun. On the other hand, such a field would have far reaching consequences on our understanding of solar activity. In this regard, we emphasize that the magnetic energy density for $\beta=10^{-3}$ at $x=0.7$ is six times the corresponding rotational energy density. Such a field may have consequences for neutrino propagation - see the review by Smirnov (1989) in these proceedings. 


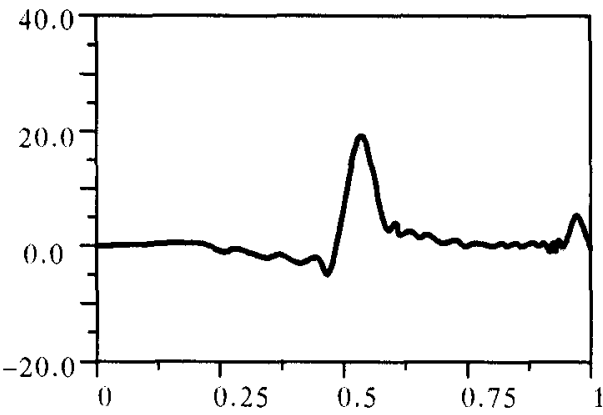

(a)

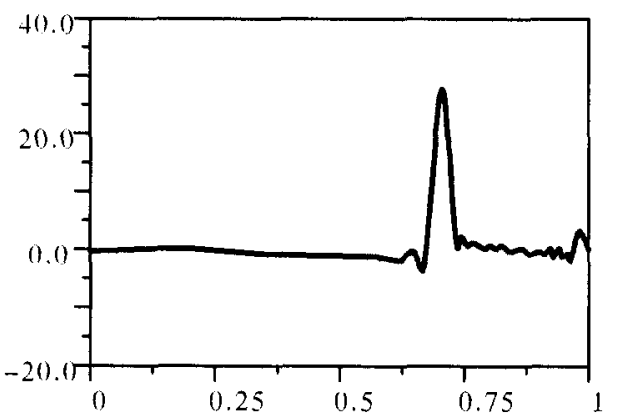

(c)



(e)

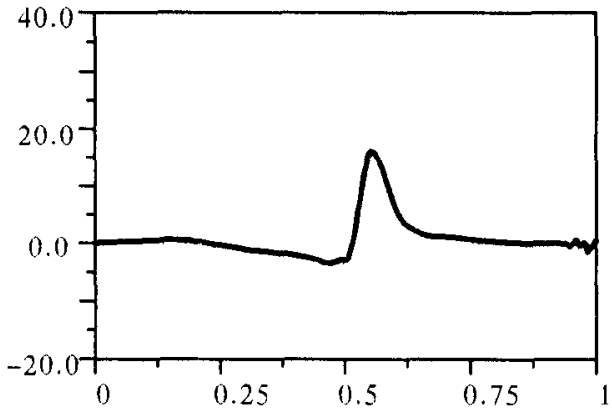

(b)



(d)

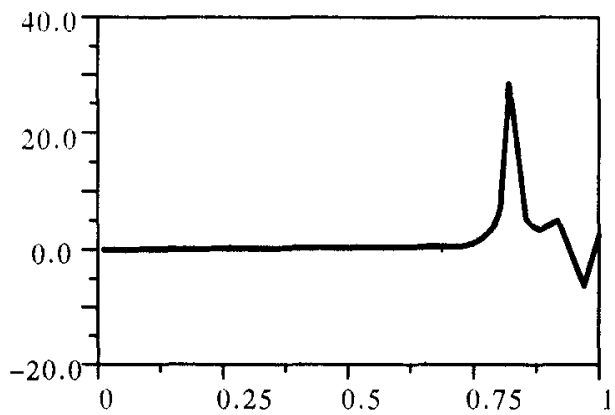

(f)

Figure 3. Unimodular Backus - Gilbert kernels,
a) $\mathrm{K}_{2}(\mathrm{x}, 0.55)$, b) $\mathrm{K}_{4}(\mathrm{x}, 0.55)$,
c) $\mathrm{K}_{2}(\mathrm{x}, 0.7)$
d) $\mathrm{K}_{4}(\mathrm{x}, 0.7)$
e) $K_{2}(x, 0.9)$,
f) $K_{4}(x, 0.9)$

vs the fractional radius. 


\section{References:}

Backus, G. and Gilbert, F. 1970, Phil. Trans Roy. Soc. London A266, 12.

Brodsky, M.A. and Vorontsov, S.V. 1988, Advances in Helio- and Asteroseismology, 137, J.Christensen-Dalsgaard and S. Fransden (Eds.), Reidel, Dordrecht, The Netherlands.

Brown, T.M., Christensen-Dalsgaard, J., Dziembowski, W.A., Goode, P.R., Gough, D.O., and Morrow, C.A.1989, Ap.J., in press.

Brown, T.M. and Morrow, C.A. 1987, Ap. J. Lett. 314, L21.

Christensen-Dalsgaard, J., Duvall, T.J., Gough, D.O., Harvey, J.W. and Rhodes, E.J. 1985, Nature 315, 378.

Christensen-Dalsgaard, J. and Shou, J. 1988, Seismology of the Sun and Sun-like Stars, 149, E.J. Rolfe (Ed.), ESA, Noordwijk, The Netherlands.

Duvall, T.J., Dziembowski, W.A., Goode, P.R., Gough, D.O., Harvey, J.W. and Leibacher, J.W. 1984, Nature 310, 22.

Duvall, T.J., Harvey, J.W. and Pomerantz, M.A. 1986, Nature 321, 500.

Dziembowski, W.A. and Goode, P.R. 1989, Ap. J, in press.

Dziembowski, W.A.,Goode, P.R., Libbrecht, K.G. 1989, Ap.J., 337, L53.

Kuhn, J.R. 1988, Ap. J. Lett. 331, L131.

Libbrecht, K.G. 1989, A p.J., 336, 1092.

Parker, E.N. 1987, The Internal Solar Angular Velocity, 289, B. Durney and S. Sofia (Eds.), Reidel, Dordrecht, The Netherlands.

Smirnov, A.Y. 1989, this volume.

Spruit, H.C. 1987, The Internal Solar Angular Velocity, 185, B. Durney and S. Sofia (Eds.), Reidel, Dordrecht, The Netherlands.

Ulrich, R.K. and Rhodes, E.J. 1983, Ap. J. 265, 551. 